\title{
Experiments and volume-of-fluid (VOF) simulations of a three-fluid dam-break
}

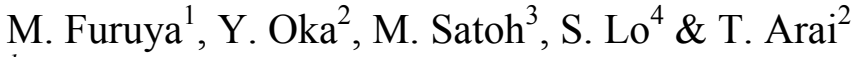 \\ ${ }^{1}$ Central Research Institute of Electric Power Industry (CRIEPI), Japan \\ ${ }^{2}$ Waseda University, Japan \\ ${ }^{3} \mathrm{CD}$ Adapco, Japan \\ ${ }^{4} \mathrm{CD}$ Adapco, $U K$
}

\begin{abstract}
Three-fluid dam-break experiments were conducted to observe the mixing and stratification processes of three immiscible fluids (two-liquids and one gas) by gravity. Two liquids (silicone oil and salt water) were separated with a vertical wall and filled in a rectangle container. Fluid motions are visualized by four sets of video cameras which are synchronized with each other. Parametric study reviles the effects of two key fluid properties: kinematic viscosity (or molecular weight) for silicone oil and density (or concentration) for sodium chloride aqueous water. After the withdrawal of a vertical partition plate, two liquids intersected earlier at the center, while the fluids stuck on the walls. The kinematic viscosity and density difference affect the three-dimensional mixing and stratification processes significantly. These visual databases are suitable for a code validation on the interfacial phenomena. Computational multi-phase fluid dynamics analysis was conducted with Star CCM+ version 8.04. The numerical results agree with experimental results accordingly for all those key parameters. The physics behind the contact angle and interfacial tensions are thoroughly investigated parametrically.

Keywords: gravity-driven stratification, injection, mixture, phase separation, liquid-liquid, flow visualization, VOF, validation.
\end{abstract}

\section{Introduction}

Mixing and stratification processes of two (or more) immiscible fluids are of importance to determine dispersion and homogeneity efficiencies in the chemical and food-processing industries, and bioengineering. During such a mixing 
process, forces acting on the fluid interface change the shape of the interface and vice versa.

A simple example is a dam-break problem. Typical fluid combination is water and air. The main focus concerned the wave front velocity and the flow depth. Chanson [1] derived a simplified solution to determine the wave tip profile based on the St. Venant equations. Ozmen-Cagatay and Kocaman [2] compared the solutions of the shallow-water equations and the Reynolds averaged Navier-Stokes (RANS) equation and concluded that the simplified solution can predict RANS numerical results.

As computer memories and performances increased significantly, threedimensional simulations became possible. LaRocque et al. [3] compared the numerical volume-of-fluid (VOF) simulations with particle image velocimetry results and found the velocity profiles between the experimental and VOF results were in good agreement. They set the wall boundaries slip free, since they focus on the inertia-driven velocity profile.

The wall and interfacial shears are of importance in a small-scale mixing and stratification processes. In order to gain insight into interfacial shear in the mixing and stratification processes, this study focuses on the interfacial motions between two immiscible liquids with a free surface (two-liquids and one gas), namely the three-fluid dam problem. The experiments for the three-fluid dam were dedicated to visualize three-dimensional interfacial wave motions between two immiscible liquids.

This paper addresses visual observation and the numerical VOF simulation of mixing and stratification processes during three-fluid dam-break. The numerical VOF simulations were extended to include a sensitivity study of two key parameters for the mixing and stratification processes: contact angle and interfacial tension.

\section{Experimental setup}

Figure 1 shows schematic of the test vessel. The internal dimensions are $96 \mathrm{~mm}$ (width), $71 \mathrm{~mm}$ (length) and $102 \mathrm{~mm}$ (height). A partition plate was inserted vertically, which separates two liquids at the center of vessel width. The thickness of the partition plate is $0.5 \mathrm{~mm}$. The two liquids were filled in the vessel at a level of $45 \mathrm{~mm}$ from the bottom. The test liquids are silicone oil and sodium chloride aqueous solution. In order to gain visibility, the sodium chloride aqueous solution was colored to blue by adding 3,7-bis (Dimethylamino)phenothiazin-5-ium chloride (hereinafter methylene blue). The methylene blue concentration is too small $(7 \mathrm{ppm})$ to affect the fluid dynamic properties.

Experimental parameters are concentration of the salt water and kinematic viscosity of the silicone oil. The density difference between liquids can change by selecting the different concentration of the salt water. We chose $10 \mathrm{wt} \%$ and $20 \mathrm{wt} \%$ of sodium chloride aqueous solution as the hydrodynamic properties are well investigated for these concentration. The silicone oil used in this study is linear polydimethylsiloxane polymers, which is a product of Momentive Performance Materials Japan LLC. The kinematic viscosity of the silicone oil, $v$ 
is selected as 200,1000 and $5000 \mathrm{~mm}^{2} / \mathrm{s}$, which correspond to the product names of Element14 PDMS 200-JC, 1000-JC and 5000-JC, respectively [4]. Disturbance of withdrawing the partition plate is negligibly small for such a high viscosity oil. Within these viscosity range, wave motions can attain better visual image of dam-break waves and does not consume a long duration.

The dam-break waves were visualized with four synchronized high-resolution video-cameras recorded from four different angles: top, front, right and left sides. The frame rate is 30 frames.

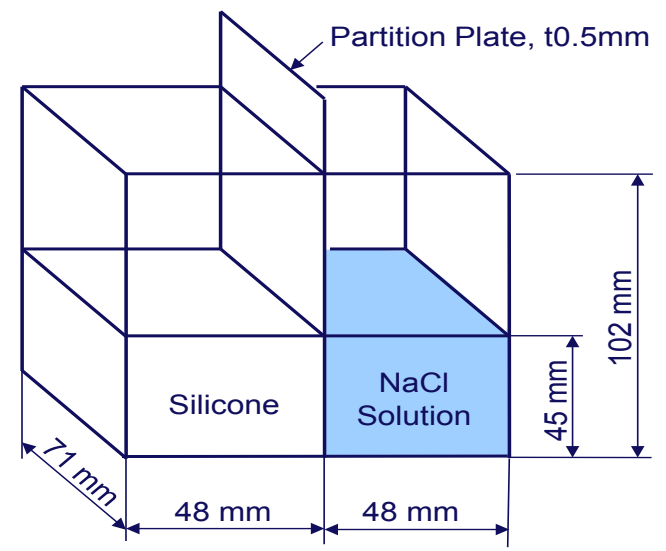

Figure 1: Schematic of test vessel. The vertical partition plate separates silicone oil from sodium chloride solution.

\section{Numerical scheme}

The commercial code StarCCM+, version 8.04 was used to simulate dam-break waves. The VOF method as developed by Muzaferija and Peric [5] was applied. Backbill et al. [6] described the interfacial tension model as a function of VOF, which is implemented in the code. The simulation mesh consists of 89,856 cells of hexahedra mesh. The mesh width is uniform and approximately $2 \mathrm{~mm}$ in all direction for simplicity. In order to ensure results with a sufficient level of accuracy, a time-step size of $0.01 \mathrm{~s}$ is used throughout, with a maximum run-time of $100 \mathrm{~s}$. As the number of inner iteration per each full iteration is limited to 5 in order to minimize computation time, this equates to approximately 50,000 iterations per simulation. The simulation used a first-order implicit unsteady and second-order special discretization scheme. The energy equation is turned off.

All the fluids (salt water, silicone oil and air) are treated as incompressible. Table 1 summarized the fluid property of silicone oil [4] and used in the analysis. Although interfacial tension between the silicone oil and the salt water remains unknown, the value between the silicone oil and water, $0.024 \mathrm{~N} / \mathrm{m}[6]$ is assumed, since surface tension of the salt water is approximately the same as that of water.

The numerical simulations starts from stagnant condition without the partition plate. All the walls set non-slip. For the sensitivity study, contact angle of the salt 
water against the silicone oil is assumed to be 30, 90 and 150 degrees. In addition, interfacial tension between the oil and the salt water is assumed to be zero and twice as high as estimated value.

Table 1: Fluid property of silicone Oil [6].

\begin{tabular}{ccccc}
\hline Product No. & TSF451 & -200 & -1000 & -5000 \\
\hline \hline Kinematic viscosity & $\mathrm{mm}^{2} / \mathrm{s}$ & 200 & 1000 & 5000 \\
\hline Density & $\mathrm{kg} / \mathrm{m}^{3}$ & 969 & 971 & 975 \\
\hline Surfce tension & $\mathrm{mN} / \mathrm{m}$ & 20.9 & 21.1 & 21.3 \\
\hline Refractive index & - & 1.403 & 1.404 & 1.404 \\
\hline
\end{tabular}

\section{Results and discussions}

Figure 2 shows a successive stages of dam-break waves of silicone oil, $v=5000$ $\mathrm{mm}^{2} / \mathrm{s}$ vs. $10 \mathrm{wt} \%$ sodium chloride aqueous solution. Video frames are taken in four different angles (top, front, right and left) and are shown in each column. The time on the left side indicates the elapsed time after the partition plate was withdrawn. The silicone oil is colored to blue for the better visibility.

The free surface of both liquids to air remained at the same height, since the viscosity of silicone oil is relatively high. At $2 \mathrm{~s}$, the salt water (denser liquid) moves underneath the silicone oil, pushing the oil upwards, and at this instant of time almost only the silicon oil is in a contact with air. One may notice a distinct change in curvature of interface on the vertical centerline of the front view. The top view shows the fact that the fluids stick to the wall while the distant fluids moves further.

Figure 3 shows a successive stages of dam-break waves of silicone oil, $v=5000 \mathrm{~mm}^{2} / \mathrm{s} v s .20 \mathrm{wt} \%$ sodium chloride aqueous solution. The density difference between $20 \mathrm{wt} \%$ salt water and the silicone oil is approximately twice as large as that between $10 \mathrm{wt} \%$ salt water and the silicone oil. The change in the wave shape is approximately twice as fast; $2 \mathrm{~s}$ and $4 \mathrm{~s}$ for $20 \mathrm{wt} \%$ salt water in the figure 3 resembles $4 \mathrm{~s}$ and $8 \mathrm{~s}$ for $10 \mathrm{wt} \%$ salt water in the figure 2 , respectively.

Figures 4 and 5 show a successive stages of dam-break waves of silicone oil, $v=200 \mathrm{~mm}^{2} / \mathrm{s} v s .10 \mathrm{wt} \%$ and $20 \mathrm{wt} \%$ sodium chloride aqueous solutions, respectively. As the kinematic viscosity is twenty-five fold smaller, the change in the wave shape is much faster. Otherwise, the dam-break waves are similar to those for $v=5000 \mathrm{~mm}^{2} / \mathrm{s}$. Experiments are conducted by using silicone oil with the kinematic viscosity of $1000 \mathrm{~mm}^{2} / \mathrm{s}$, though the behavior falls in those of between $200 \mathrm{~mm}^{2} / \mathrm{s}$ and $5000 \mathrm{~mm}^{2} / \mathrm{s}$. These figures are omitted due to space limitations. 


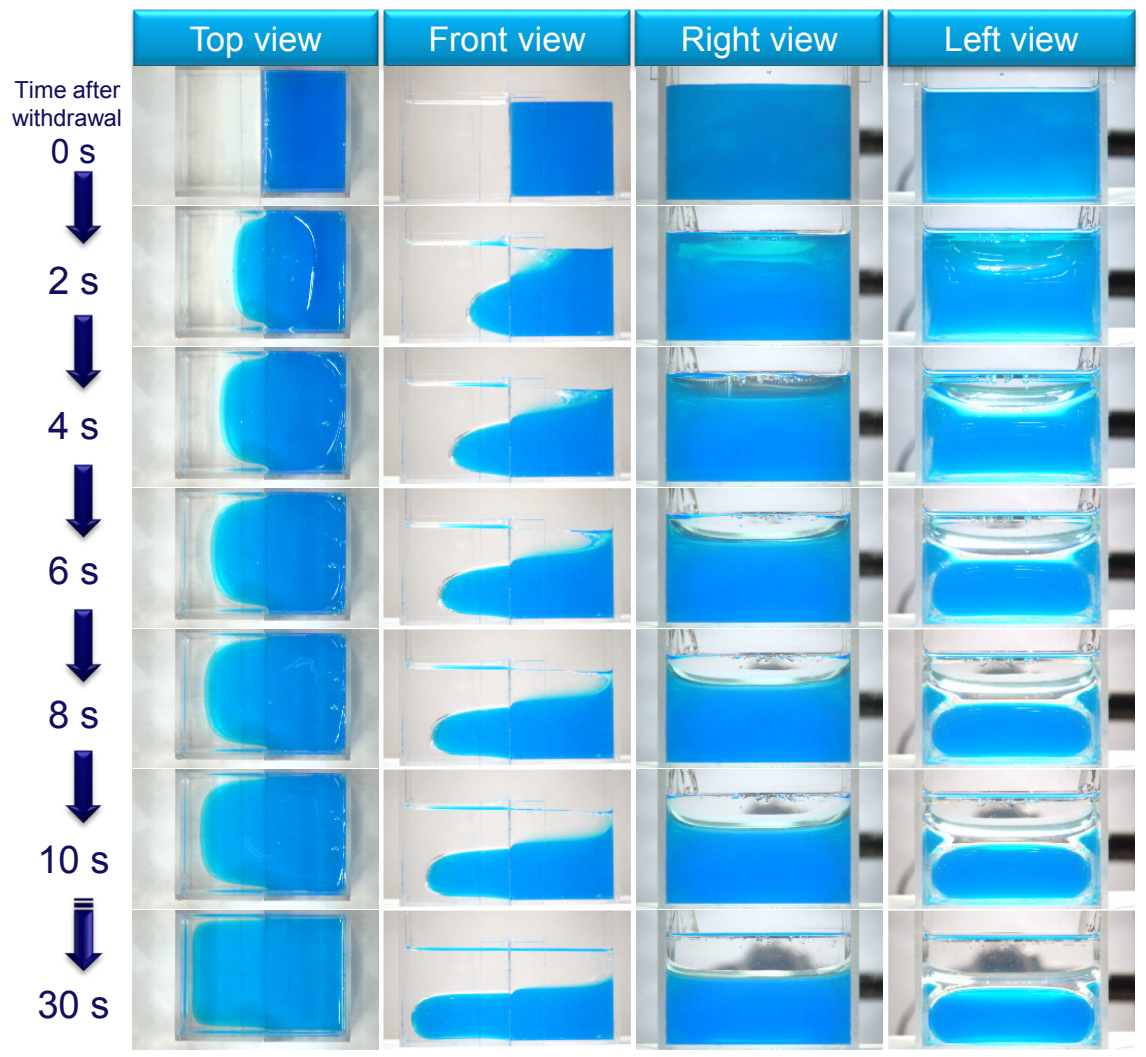

Figure 2: Successive stages of dam-break waves of silicone oil, $v=5000$ $\mathrm{mm}^{2} / \mathrm{s}$ (initially left side and transparent) $v s .10 \mathrm{wt} \%$ sodium chloride aqueous solution (initially right side and colored blue with 7 ppm MB). 


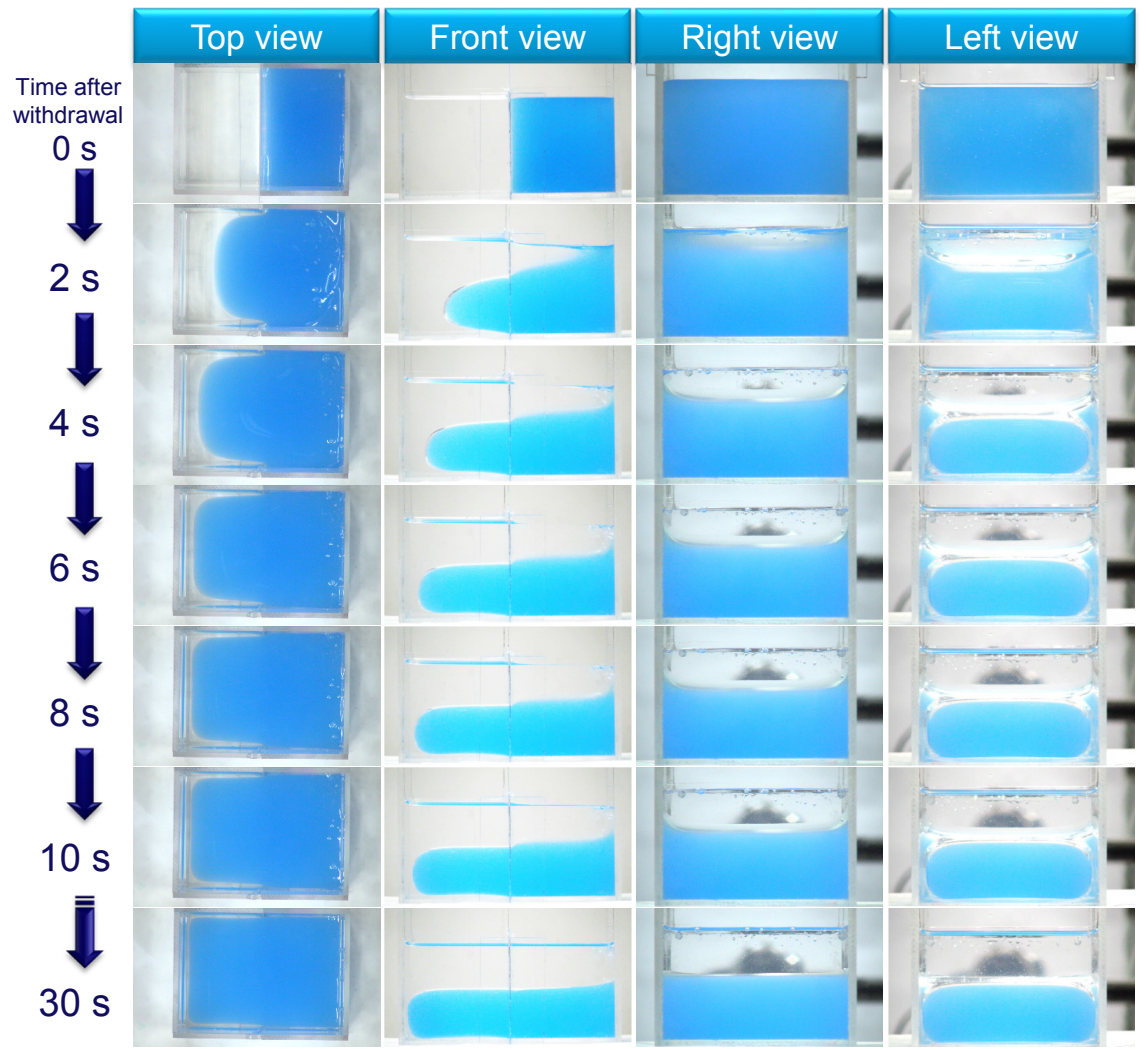

Figure 3: Successive stages of dam-break waves of silicone oil, $v=5000$ $\mathrm{mm}^{2} / \mathrm{s}$ (initially left side and transparent) vs. $20 \mathrm{wt} \%$ sodium chloride aqueous solution (initially right side and colored blue with 7 ppm MB). 


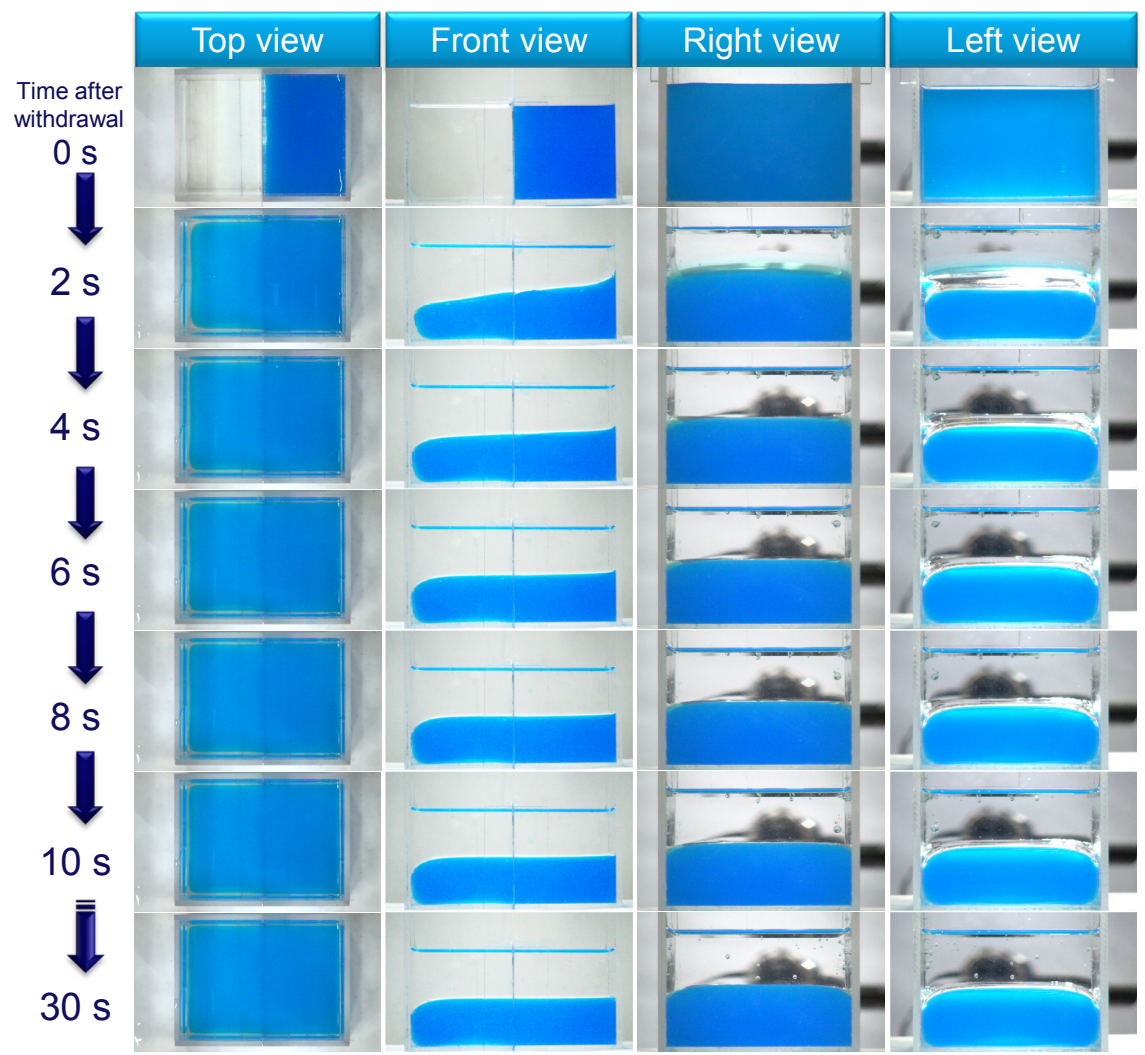

Figure 4: Successive stages of dam-break waves of silicone oil, $v=200$ $\mathrm{mm}^{2} / \mathrm{s}$ (initially left side and transparent) vs. $10 \mathrm{wt} \%$ sodium chloride aqueous solution (initially right side and colored blue with 7 ppm MB). 


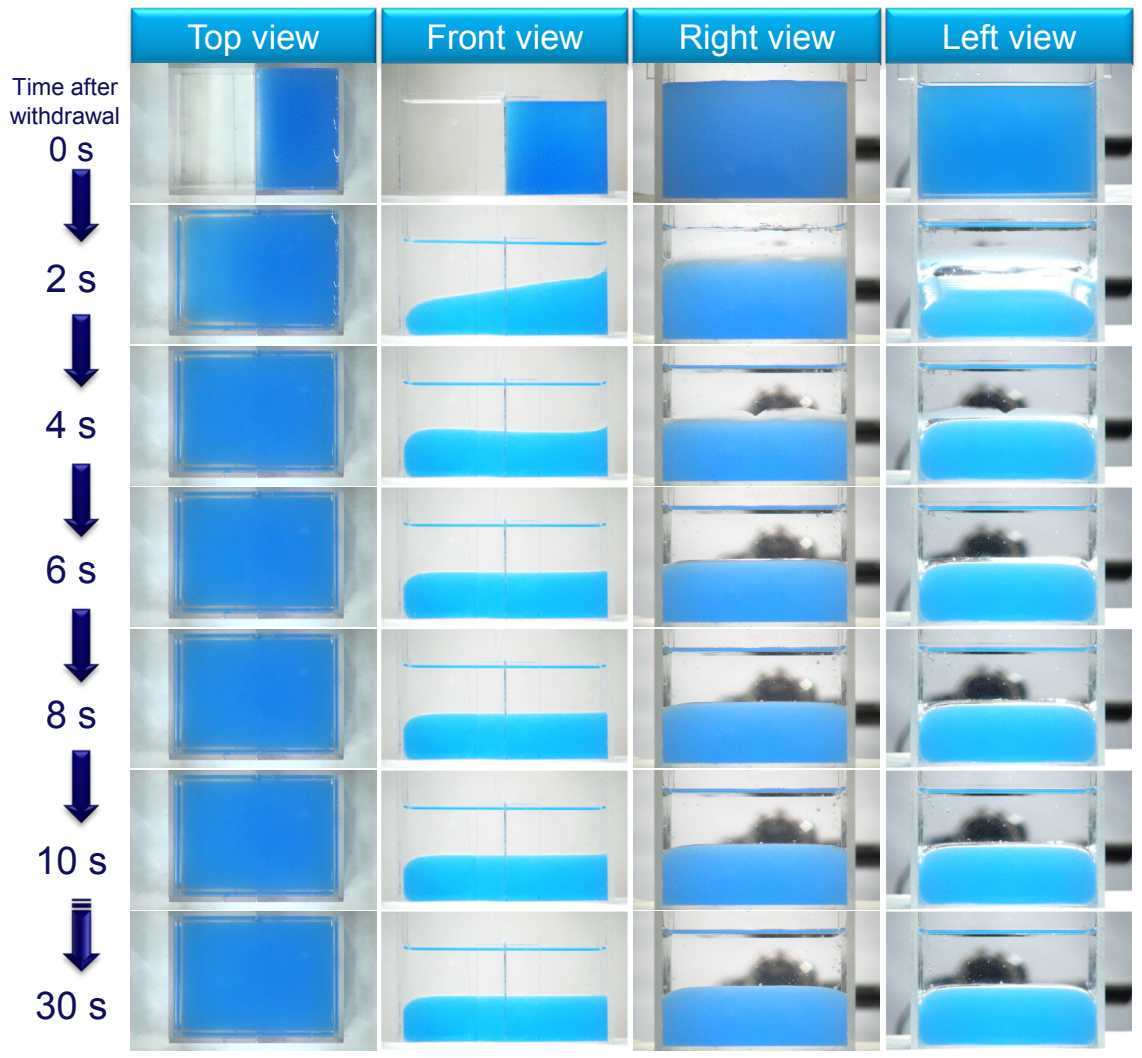

Figure 5: Successive stages of dam-break waves of silicone oil, $v=200$ $\mathrm{mm}^{2} / \mathrm{s}$ (initially left side and transparent) vs. $20 \mathrm{wt} \%$ sodium chloride aqueous solution (initially right side and colored blue with 7 ppm MB). 
Figure 6 shows comparison between the experimental results and VOF simulation of dam-break waves of silicone oil, $v=5000 \mathrm{~mm}^{2} / \mathrm{s} v s .10 \mathrm{wt} \%$ sodium chloride aqueous solution. The contact angle for the VOF simulation is 90 degrees. The VOF simulation reproduces the dam-break waves in shapes and speed of change. The free surface of both liquids to air remained at the same height.

Figure 7 shows an effect of contact angle for the dam-break waves of silicone oil, $v=5000 \mathrm{~mm}^{2} / \mathrm{s} v \mathrm{~s} .10 \mathrm{wt} \%$ sodium chloride aqueous solution. The separate effect experiment indicates the contact angle of $10 \mathrm{wt} \%$ salt water against the silicone oil was 90 degrees or little larger. The figure 7 (b) resembles, therefore experimental results the better. The waves advances on the wall for the contact angle of 30 degrees, while the interface stick to the wall for that of 150 degrees. The VOF simulation clearly represents the specified contact angle differences in the shape of interface and its angle to the front and back walls, and floor. On the contrary, these differences do not change the position of leading edge significantly as indicated by the arrow and scale at the top left in each figure.

Figure 8 shows an effect of interfacial tension on the dam-break waves of silicone oil, $v=5000 \mathrm{~mm}^{2} / \mathrm{s} v s .10 \mathrm{wt} \%$ sodium chloride aqueous solution. The interface deforms more significantly by neglecting interfacial tension $\left(\sigma_{i}=0\right)$ as shown in the figure 8 (a). The interfacial area becomes smaller as doubled interfacial tension $\left(\sigma_{i}=\sigma_{\text {silicone,water }}\right)$ as shown in the figure $8(\mathrm{c})$. Increasing the interfacial tension decreases the advancing speed of leading edge as indicated by an arrow and a scale at the top left in each figure. The results suggest that the interfacial tension affect the speed of dam-break wave motion in such a small scale.

\section{Concluding remarks}

Three-fluid dam-break experiments were conducted to observe mixing and stratification processes of three immiscible fluids by gravity. The experimental database were established for the code validation in terms of two key liquid properties: kinematic viscosity and density difference. After withdrawal of a vertical partition plate, two liquids intersects earlier at the center, while the liquids stick on the walls. The VOF simulations predicts the overall dam-break wave motions: three-dimensional mixing and stratification processes for these key parameters. Physics behind the contact angle and interfacial tensions are thoroughly investigated parametrically. The VOF simulations indicates that the interfacial tension affect the speed of dam-break wave motion when the wall spacing is relatively small. 


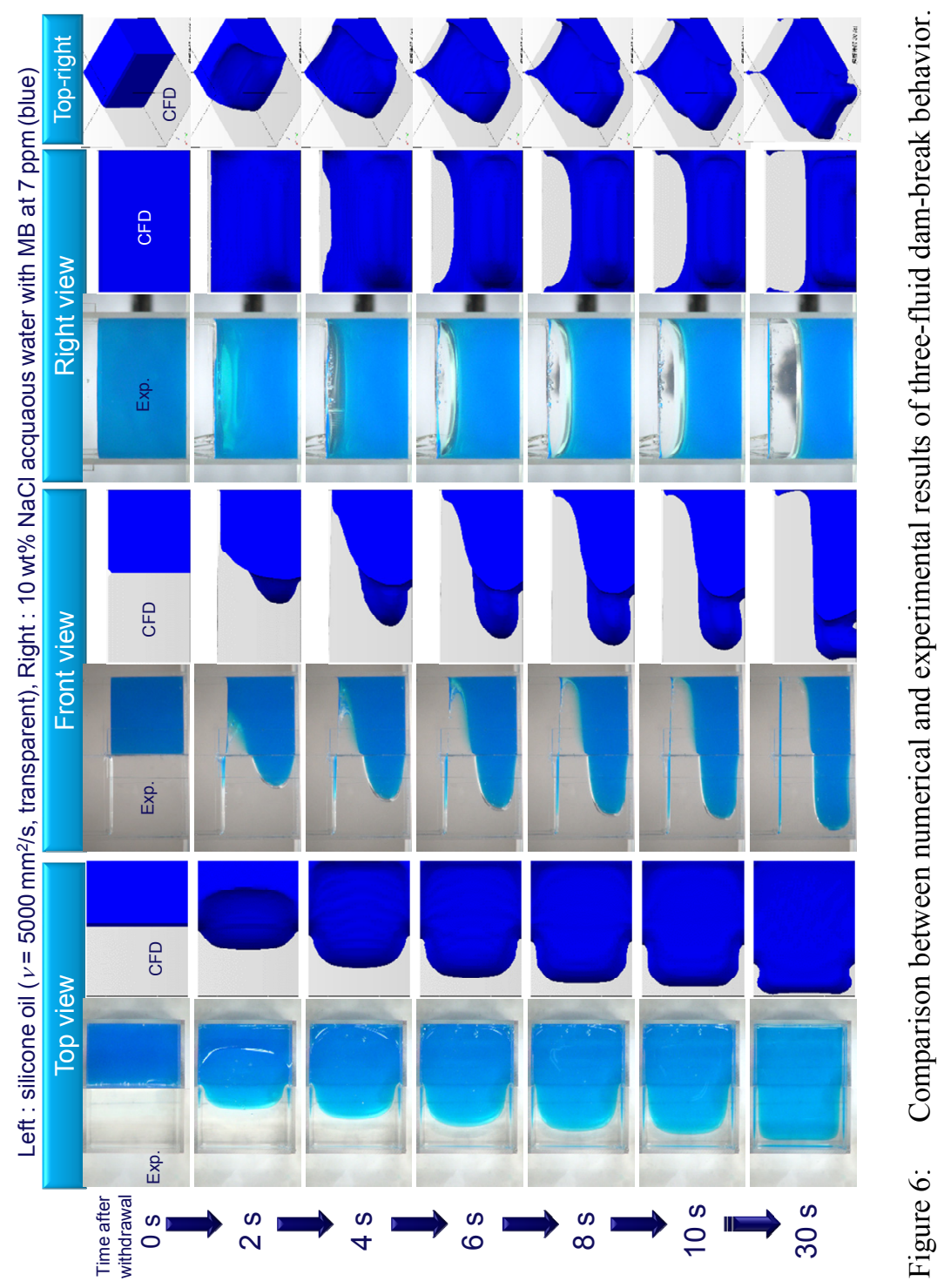



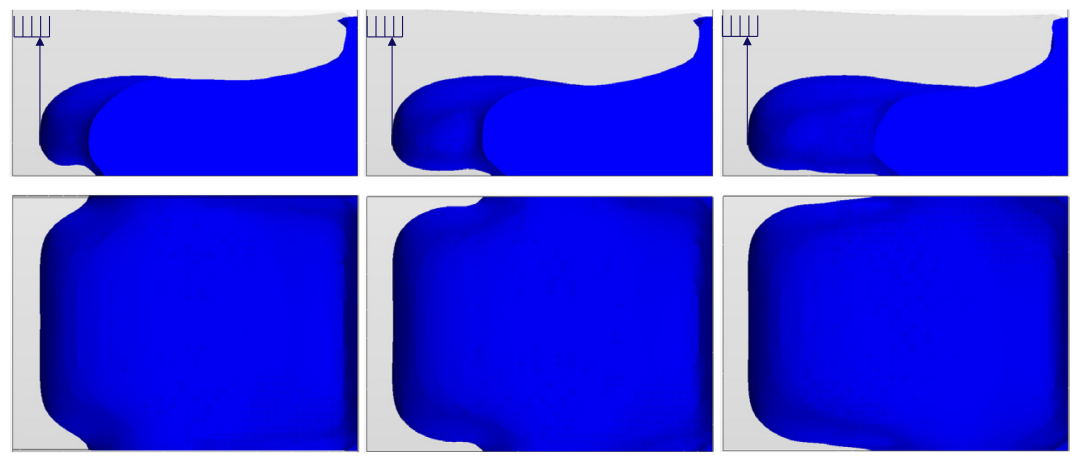

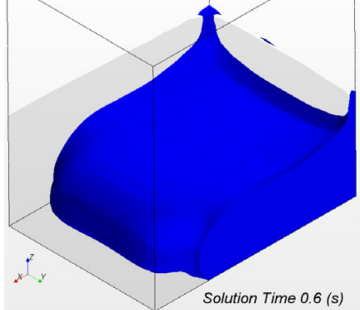

(a) Contact angle $30^{\circ}$

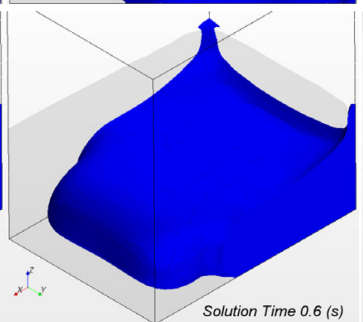

(b) Contact angle $90^{\circ}$

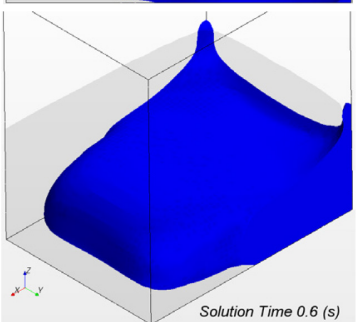

(c) Contact angle $150^{\circ}$

Figure 7: Effect of contact angle on the leading edge position.

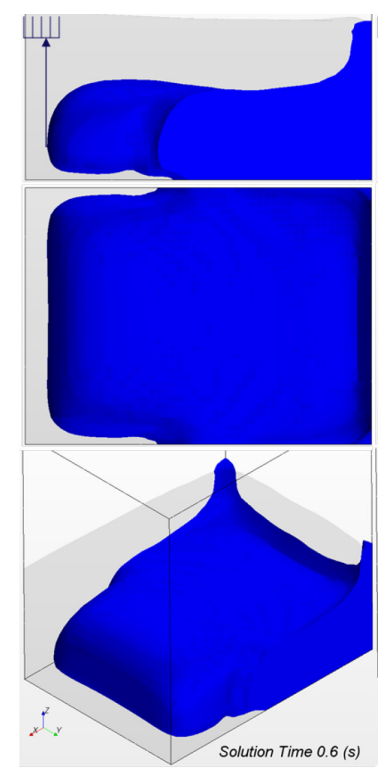

(a) $\sigma_{i}=0$
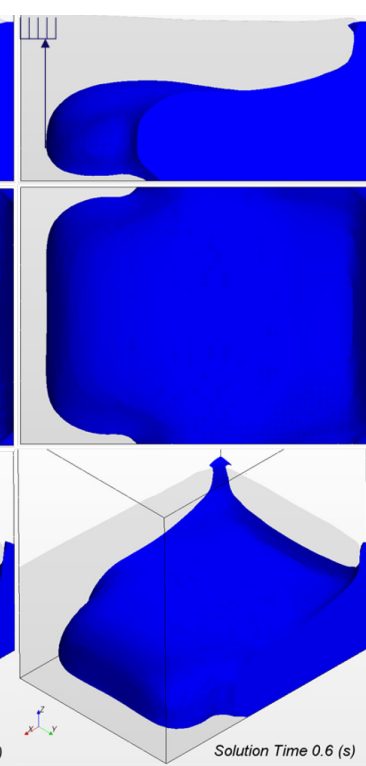

(b) $\sigma_{i}=\sigma_{\text {silicone,water }}$
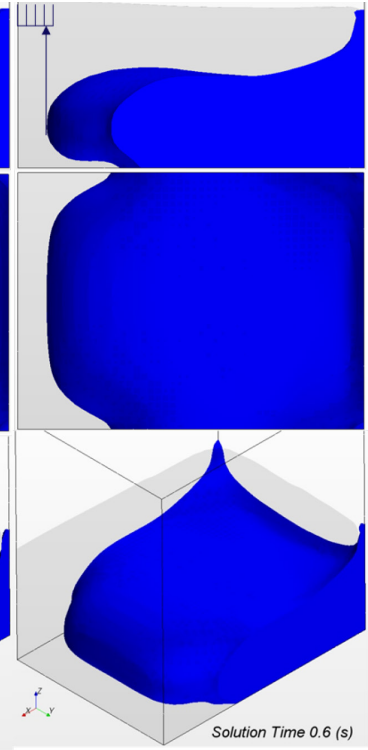

(c) $\sigma_{i}=2 \sigma_{\text {silicone, water }}$

Figure 8: Effect of interfacial tension on the leading edge position. 


\section{Acknowledgements}

A part of this study is the result of "Mechanistic study of melt behavior in lower RPV head" carried out under the Strategic Promotion Program for Basic Nuclear Research by the Ministry of Education, Culture, Sports, Science and Technology of Japan. The authors are grateful for withdrawing the partition plate smoothly by Mr. Tatsumi Ikeda. Thanks are extended to Dr. Samir Muzaferija, Dr. Muneki Sakata and Dr. Chika Kakegawa of CD-Adapco for the valuable comments on the StarCCM+ simulation.

\section{References}

[1] Chanson, H. "Application of the method of characteristics to the dam break wave problem,” J. Hydraulic Res. 47(1), pp. 41-49, 2009.

[2] Ozmen-Cagatay, H., Kocaman, S., "Dam-break flows during initial stage using SWE and RANS approaches," J. Hydraulic Res. 48(5), pp. 603-611, 2010.

[3] LaRocque, L. A., Imran, J., Chaudhry, M. H., "Experimental and Numerical Investigations of Two-Dimensional Dam-Break Flows", J. Hydraulic Engineering, 139(6), pp. 569-579, 2013.

[4] Element14 PDMS Prodact data, MK-IN-1207031, Momentive Performance Materials Japan LLC, 2012.

[5] Muzaferija, S., Peric, M., "Computation of free surface flows using interface-tracking and interface-capturing methods," chapter 2 in O. Mahrenholtz and M. Markiewicz (eds.), Nonlinear Water Wave Interaction, pp. 59-100, WIT Press, Southampton, 1999.

[6] Backbill, J. U., Kothe, D. B., Zemach, C., "A Continuum Method for Modeling Surface Tension,” J. Computational Physics, 100, pp. 335-354, 1992.

[7] Than, P., Preziosi, L., Joseph, D. D., Arney, M., "Measurement of Interfacial Tension between Immiscible Liquids with the Spinning Rod Tensiometer," J. Colloid and Interface Science, 124(2), pp. 552-559, 1988. 